\title{
An Enzyme-Mediated Amplification Strategy Enables Detection of $\beta$-Lactamase Activity Directly in Unprocessed Clinical Samples for Phenotypic Detection of $\beta$-Lactam Resistance
}

\author{
Tara R. deBoer ${ }^{a}$, Nicole J. Tarlton ${ }^{b}$, Reina Yamaji ${ }^{b}$, Sheila Adams-Sapper ${ }^{b}$, Tiffany Z. Wu ${ }^{b}$, \\ Santanu Maitya ${ }^{a}$, Giri K. Vesgesna ${ }^{a}$, Corinne M. Sadlowski ${ }^{\mathrm{a}}$, Peter DePaola IV ${ }^{\mathrm{a}}$, Lee W. \\ Riley $^{b}$, and Niren Murthy ${ }^{a}$ \\ ${ }^{a}$ Department of Bioengineering, University of California, Berkeley University Avenue, Berkeley, CA \\ 94720 (USA) \\ bDepartment of Public Health, University of California, Berkeley University Avenue, Berkeley, CA \\ 94720 (USA)
}

\begin{abstract}
Biochemical assays that can identify $\beta$-lactamase activity directly from patient samples have the potential to significantly improve the treatment of bacterial infections. However, current $\beta$ lactamase probes do not have the sensitivity needed to measure $\beta$-lactam resistance directly from patient samples. Here, we report the development of an instrument-free signal amplification technology, DETECT, that connects the activity of two enzymes in series to effectively amplify the activity of $\beta$-lactamase 40000 -fold, compared to the standard $\beta$-lactamase probe nitrocefin.
\end{abstract}

\section{Keywords}

amplification; antimicrobial resistance; diagnostics; beta-lactamases; point-of-care diagnosis

\begin{abstract}
$\beta$-Lactamases are enzymes adapted by bacterial pathogens to evade the bactericidal capacity of $\beta$-lactam antibiotics, through hydrolysis of the $\beta$-lactam pharmacophore of these therapeutic agents. ${ }^{[1]} \beta$-Lactamases remain an important diagnostic target because they represent a ubiquitous resistance mechanism that significantly complicates the treatment of a wide range of bacterial infections. Diagnostic tests that can rapidly detect the presence of these enzymes directly in patient samples remain an unmet medical need. ${ }^{[2,3]}$

The lack of diagnostic options for rapidly revealing resistance biomarkers forces physicians to treat patients empirically at the time of care, then wait days to receive clinical information confirming or contradicting their selected treatment. ${ }^{[4,5]}$ Because $\beta$-lactam antibiotics are a predominant class of agents used to treat infections caused by both Gram-positive and Gram-negative bacteria, the presence of $\beta$-lactamases in bacterial pathogens can have a
\end{abstract}

Conflict of Interest

T.d.B. and N.J.T. are founders of a company that has been established based on this work.

Supporting information and the ORCID identification numbers for the authors of this article can be found under https://doi.org/ $10.1002 /$ cbic. 201800443. 
significant impact on treatment efficacy. Therefore, biochemical approaches that detect the activity of these enzymes represent an attractive strategy that can provide simple, colorimetric, real-time detection of $\beta$-lactamase activity. ${ }^{[6]}$

Unfortunately, sensitivity limitations have prevented the translation of biochemical assays into viable point-of-care (POC) diagnostic tests. Although bacterial concentrations in clinical samples are low, and can be in the range of 10 to $10^{5}$ colony forming units per $\mathrm{mL}$ $\left(\mathrm{CFU} \mathrm{mL}{ }^{-1}\right.$ ) depending on the type of sample, current commercial colorimetric biochemical assays require $>10^{7} \mathrm{CFUmL}^{-1}$ to detect $\beta$-lactamase activity. ${ }^{[7,8]}$ Therefore, the implementation of amplification strategies to circumvent the sensitivity limitations of current colorimetric biochemical assays would significantly expand the scope and impact of biochemical-based tests, and support their potential for use as POC diagnostics.

Herein, we report the development of an instrument-free amplification strategy, termed DETECT, that effectively enhances the sensitivity limit for detecting $\beta$-lactamase activity by linking the $\beta$-lactamase in series with a secondary enzyme amplifier, papain. DETECT is a four-component system composed of: 1 ) the target enzyme, $\beta$-lactamase (E1); 2 ) the enzyme amplifier, papain, ${ }^{[9]}$ caged by a disulfide formed at its active-site cysteine (E2); ${ }^{[10]} 3$ ) the $\beta$ lactamase substrate, thiophenol- $\beta$-lac, which eliminates the papain trigger, thiophenol, after cleavage by $\beta$-lactamase; and 4) the papain probe, $N$-benzoyl-L-arginine- $p$-nitroaniline (BAPA), which releases the colorimetric product $p$-nitroaniline. DETECT amplifies enzymatic activity because a single $\beta$-lactamase enzyme can activate thousands of papain molecules, and each activated papain molecule can subsequently turn over thousands of its chromogenic substrate, thus enhancing the detection sensitivity by orders of magnitude relative to traditional single-enzyme assays (Figure $1 \mathrm{~A}$ ).

Thiophenol- $\beta$-lac is the chemical link between the target $\beta$-lactamase and the enzyme amplifier papain, and contains both a targeting and triggering unit. The targeting unit features a first-generation cephalosporin core containing a benzyl moiety at the $\mathrm{C} 7$ amide, analogous to the early-generation $\beta$-lactam, benzylpenicillin. Hydrolysis of thiophenol- $\beta$-lac liberates thiophenol through a 1,4-elimination cascade, and initiates the activation of disulfide-caged papain (Figure $1 \mathrm{~B}$ ). The promiscuous benzylcephalosporin scaffold enables the detection of a broad range of $\beta$-lactamases.

We first verified that thiophenol- $\beta$-lac could target diverse $\beta$-lactamases by testing DETECT with the recombinant $\beta$-lacta-mases SHV-1, CTX-M-2, CTX-M-14, CTX-M-15, CMY-2, and KPC-2, prepared as reported in the literature. ${ }^{[11]}$ The recombinant $\beta$-lactamases were incubated with the DETECT components thiophenol- $\beta$-lac (100 mM, final concentration), caged papain (6 mM, final concentration), and BAPA ( $3.3 \mathrm{~mm}$, final concentration) within the wells of a 96-well plate. After 30 min of incubation at ambient temperature, the absorbance values at $405 \mathrm{~nm}$ were collected with a microplate reader. The $405 \mathrm{~nm}$ absorbance corresponds to the presence of $p$-nitroaniline, which is produced upon activation of the DETECT system. The final absorbance values at $30 \mathrm{~min}$ are shown in Figure 2 B. Results from this study established the molecular promiscuity of thiophenol- $\beta$-lac; DETECT signal outputs were generated only in the presence of the recombinant $\beta$-lactamases, but not in control wells that contained all the DETECT components in the absence of a $\beta$-lactamase. 
Therefore, thiophenol- $\beta$-lac was validated as an effective $\beta$-lactamase-targeting probe in the DETECT system.

Subsequently, we defined the amplification capacity of the DETECT system by using the recombinant $\beta$-lactamase, TEM-1, which mediates resistance to early-generation $\beta$-lactam antibiotics such as ampicillin. The sensitivity of DETECT was compared against the standard chromogenic $\beta$-lactamase probe, nitrocefin (Figure $2 \mathrm{C}$ and D). ${ }^{[12]}$ The limit of detection (LOD) for recombinant TEM-1 was assessed by measuring the formation of the chromogenic products from DETECT or nitrocefin in a time-dependent manner, with a microplate reader (DETECT measured at $405 \mathrm{~nm}$ and nitrocefin measured at $486 \mathrm{~nm}$ ). These studies validated the amplification power of the dual-enzymatic approach of DETECTwith an LOD of $100 \mathrm{fm}$ TEM-1 compared to nitrocefin's LOD of 4 nM TEM-1—which demonstrated a 40000 -fold enhancement in sensitivity.

Next, we evaluated the ability of DETECT to identify $\beta$-lactamase activity in clinical bacterial isolates. For this analysis, we selected $\beta$-lactam-resistant Escherichia coli isolates characterized to produce a single $\beta$-lactamase, and $\beta$-lactam-susceptible $E$. coli isolates that lacked these $\beta$-lactamases. The "positive" resistant group comprised isolates SF334, SF674, and B2, which produce TEM-1, CTX-M-15, and KPC-2, respectively; the "negative" susceptible group comprised isolates SF505, SF384, and IT917. [11, 13-15]

Bacterial suspensions of the isolates were prepared from overnight broth cultures to final optical densities at $600 \mathrm{~nm}\left(\mathrm{OD}_{600}\right.$, where $\left.0.1 \mathrm{OD}=1 \times 10^{8} \mathrm{CFU} \mathrm{mL}{ }^{-1}\right)$ of $0.5,0.4,0.3,0.2$, 0.1 , and $0.05( \pm 0.001)$. The suspension prepared to an $\mathrm{OD}_{600}$ of 0.05 was then serially diluted in one-half increments to prepare samples with presumed ODs of 0.025, 0.0125, 0.00625 , and 0.003125 . Aliquots $(5 \mu \mathrm{L})$ of each whole-cell bacterial suspension were transferred to two wells of a 96-well plate, each containing BAPA and caged papain, with one well being the sample well and the other a control well. The control well contained all of the DETECT components except thiophenol- $\beta$-lac, so as to evaluate nonspecific background signal. The incubation time was initiated when thiophenol- $\beta$-lac was added to the sample wells. After $1 \mathrm{~h}$ of incubation, the absorbance values at $405 \mathrm{~nm}\left(A_{405}\right)$ were measured. The detection parameter of DETECT is denoted as the "DETECT score" hereafter, and is defined as the $A_{405}$ of the sample well minus the $A_{405}$ of the control well after $1 \mathrm{~h}$. To determine the LOD, a signal threshold value was defined for each tested bacterial concentration by taking the average DETECT score of the negative samples at the designated concentration and adding three times their standard deviation.

The LODs of the TEM-1-producing isolate SF334 (LOD SF334 $_{4}$, CTX-M-15-producing isolate SF674 ( $\left.\mathrm{LOD}_{\mathrm{SF} 674}\right)$, and $\mathrm{KPC}-2$-producing isolate $\mathrm{B} 2\left(\mathrm{LOD}_{\mathrm{B} 2}\right)$ were $1.0 \times 10^{6}, 6.3 \times$ $10^{4}$, and $1.3 \times 10^{5} \mathrm{CFU}$, respectively. Under these conditions, nitrocefin was unable to detect $\beta$-lactamase activity in any of the isolates (Figure $3 \mathrm{~A}$, inset). Therefore, a secondary LOD study was performed to determine $\mathrm{LOD}_{\mathrm{SF} 334}, \mathrm{LOD}_{\mathrm{SF} 674}$, and $\mathrm{LOD}_{\mathrm{B} 2}$ for nitrocefin; these were found to be $4 \times 10^{7}, 4 \times 10^{6}$, and $3 \times 10^{6} \mathrm{CFU}$, respectively (Figure S1 in the Supporting Information). 
The clinical impact of the observed amplification of $\beta$-lactamase activity by DETECT supported the potential utility of this system in revealing $\beta$-lactamase activity directly from urine samples, in which bacterial concentrations associated with urinary tract infections (UTIs) typically range from $10^{4}$ to $10^{6} \mathrm{CFUmL}^{-1}$. To assess this potential, we tested 40 urine samples that were collected from patients suspected of having a UTI at the outpatient clinic at the University of California, Berkeley. ${ }^{[16]}$ No personal identifiers or clinical information were obtained from the study subjects. Urine samples were tested with DETECT by transferring $50 \mu \mathrm{L}$ aliquots of a urine sample into two independent wells of a 96-well plate, one designated "sample" and the other "control". Urine samples in sample wells were incubated with all DETECT components, whereas the corresponding control wells were incubated only with caged papain and BAPA to account for nonspecific reactions that could be triggered by free thiols or proteases potentially present in urine. Samples were incubated for $30 \mathrm{~min}$ at ambient temperature, then analyzed with a microplate reader to define the DETECT score.

In this study, standard phenotypic analyses were used as the reference test method. E. coli isolated from urine samples were tested for susceptibility to $\beta$-lactams by using the earlygeneration agent, ampicillin, and following the Clinical \& Laboratory Standards Institute guidelines. Samples that tested resistant to ampicillin were defined as positive; samples that tested susceptible to ampicillin were defined as negative. Samples that did not grow bacteria were also defined as negative. The average DETECT score from samples that were considered positive was compared to the average score from samples that were considered negative. $\beta$-Lactam-resistant samples yielded an average DETECT score of 1.026 , whereas $\beta$-lactam-susceptible samples yielded an average DETECT score of 0.2990 ( $p=0.00261$, two-tailed $t$-test; Figure $4 \mathrm{~B}$ ). The threshold value of DETECT was defined as 0.616 , which was three standard deviations greater than the average DETECT score from $\beta$-lactamsusceptible samples. Therefore, samples with DETECT scores $>0.616$ were defined as "positive" (resistant to $\beta$-lactams) by DETECT, and samples with DETECT scores $<0.616$ were defined as "negative" (susceptible to $\beta$-lactams) by DETECT.

Based on this analysis, DETECT identified the absence of $\beta$-lactamase activity in 22 of 23 urine samples (Table S1). In addition, DETECT identified eight of 11 urine samples containing bacteria with resistance to early-generation $\beta$-lactams (97\% specificity, 73\% sensitivity). Genotypic analysis of the $11 \beta$-lactam-resistant E. coli revealed that ten (91\%) of the isolates produced TEM-1 $\beta$-lactamases and three (27\%) isolates produced a CTX-M $\beta$-lactamase (from CTX-M-g1 or CTX-M-g9). ${ }^{[17]}$ Two of three resistant urine samples that were missed by DETECT were later characterized to contain TEM-1-producing E. coli; the third sample was found to contain a TEM-1/CTX-M-g9-producing E. coli (likely CTX$\mathrm{M}-14$, Table S1). The E. coli isolated from these three false-negative and one false-positive urine sample were subsequently analyzed by DETECT and found to be correctly positive or correctly negative, respectively (Figure S2). We speculate that the original incorrect results were likely due to very low $\mathrm{CFUmL}^{-1}$ concentrations in the false-negative urine samples, or a very high $\mathrm{CFUmL}^{-1}$ concentration in the false-positive urine sample.

Overall, the instrument-free amplification capacity of DETECT afforded a significant enhancement in the ability to detect $\beta$-lactamases. This enabled the identification of $\beta$ - 
lactamase activity directly from patient urine samples, without any need for culturing, sample centrifugation/concentration, or cell lysis. One possible issue with DETECT is the potential for nonspecific triggering of the system by thiols that could be present in bacterial suspensions or urine. However, by evaluating the DETECT score instead of the raw absorbance reading, these nonspecific signals were accounted for in the analysis.

Furthermore, the quantitative absorbance-based signal output feature is amenable to numerous device formats, ranging from smart digital devices to paper-based tests. As such, DETECT represents an innovative amplification solution that could be applied as a diagnostic test to detect $\beta$-lactamase activity in patient samples, to direct antimicrobial therapy in a POC fashion.

\section{Supplementary Material}

Refer to Web version on PubMed Central for supplementary material.

\section{Acknowledgements}

We thank the National Institutes of Health (NIH R01 EB 023776 and NIH RO1 AI117064) for supporting this work. T.d.B. was also supported by NIH grant AI 117064-02 S1.

\section{References}

[1]. "The Mechanisms of Resistance to $\beta$-Lactam Antibiotics", King DT, Sobhanifar S, Strynadka NCJ in Handbook of Antimicrobial Resistance (Eds.: Gotte M, Berghuis A, Matlashewski G, Wainberg M, Sheppard D), Springer, New York, 2017, pp. 177.

[2]. Blaschke AJ, Hersh AL, Beekmann SE, Ince D, Polgreen PM, Hanson KE, Diagn. Microbiol. Infect. Dis 2015, 81, 57-59. [PubMed: 25456043]

[3]. Galar A, Leiva J, Espinosa M, Guillen-Grima F, Hernaez S, Yuste JR, J. Infect 2012, 65, 302-309. [PubMed: 22728132]

[4]. Sugden R, Kelly R, Davies S, Nat. Microbiol 2016, 1, 16187. [PubMed: 27670123]

[5]. Perez KK, Olsen RJ, Musick WL, Cernoch PL, Davis JR, Peterson LE, Musser JM, J. Infect 2014, 69, 216-225. [PubMed: 24841135]

[6]. Shinde S, Gupta R, Raut SS, Nataraj G, Mehta PR, J. Lab. Physicians 2017, 2, 100-103.

[7]. Rudresh SM, Ravi GS, Sunitha L, Hajira SN, Kalaiarasan E, Harish BN, J. Lab. Physicians 2017, 4, 303-307.

[8]. Renvoise A, Decre D, Amarsy-Guerle R, Huang T-D, Jost C, Podgla-jen I, Raskine L, Genel N, Bogaerts P, Jarlier V, Arlet G, J. Clin. Microbiol 2013, 51, 4012-4017. [PubMed: 24068012]

[9]. Singh R, Blattler WA, Collinson AR, Anal. Biochem 1993, 213, 49-56. [PubMed: 8238881]

[10]. Brocklehurst K, Little G, Biochem. J 1973, 133, 67-80. [PubMed: 4721623]

[11]. Tarlton NJ, Satoorian TS, Panchal A, Borges CA, Geisberg M, Riley LW, J. Microbiol. Methods 2018, 144, 37-43. [PubMed: 28970054]

[12]. O'Callaghan CH, Morris A, Kirby SM, Shingler AH, Antimicrob. Agents Chemother 1972, 1, 283-288. [PubMed: 4208895]

[13]. Adams-Sapper S, Diep BA, Perdreau-Remington F, Riley LW, Antimi-crob. Agents Chemother 2013, 57, 490-497.

[14]. Adams-Sapper S, Nolen S, Donzelli GF, Lal M, Chen K, Justo da Silva LH, Moreira BM, Riley LW, Antimicrob. Agents Chemother 2015, 59, 3281-3289. [PubMed: 25801565]

[15]. Smith SP, Manges AR, Riley LW, Clin. Infect. Dis 2008, 46, 689-695. [PubMed: 18230040]

[16]. Yamaji R, Rubin J, Thys E, Friedman CR, Riley LW, J. Clin. Microbiol 2018, 56, e01834-17. [PubMed: 29436416] 
[17]. Dallenne C, Da Costa A, Decre D, Favier C, Arlet G, J. Antimicrob. Che-mother 2010, 65, 490495. 
A)

DETECT: Dual-Enzyme Amplification

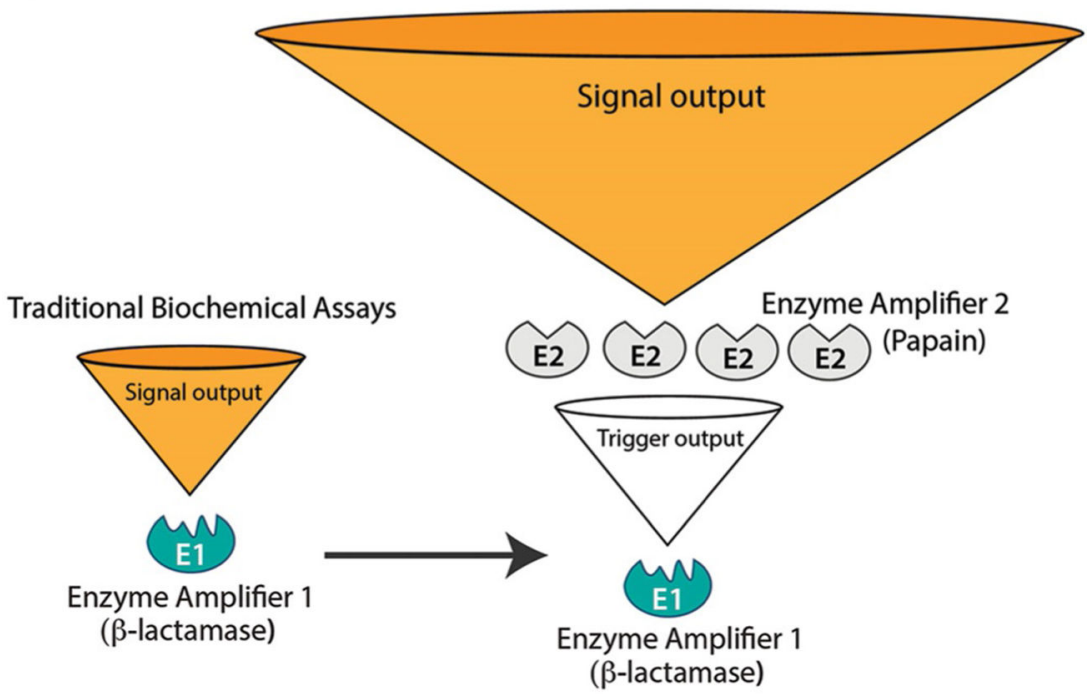

B)

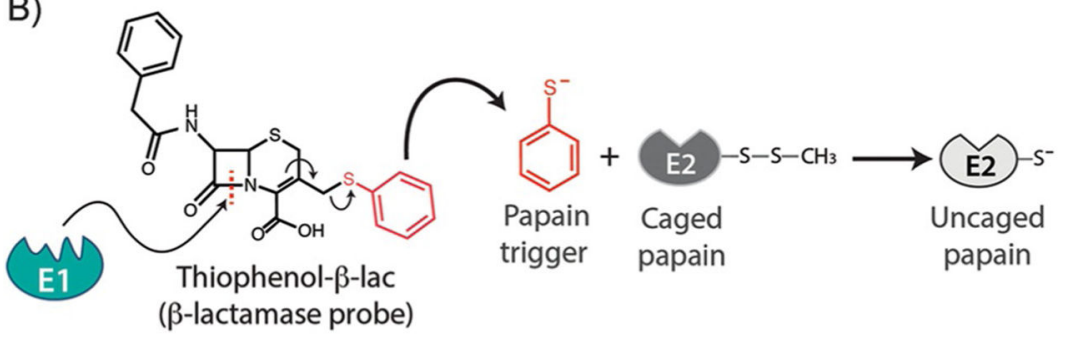

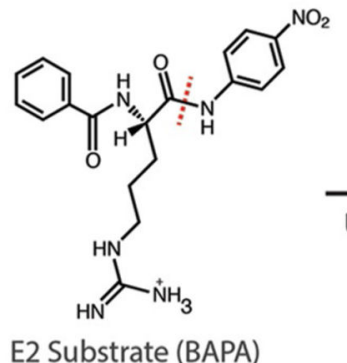
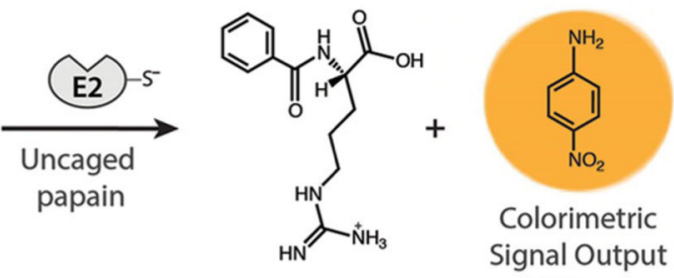

$(405 \mathrm{~nm})$

Figure 1.

A) Pictorial comparison of the amplification capacity of DETECT relative to traditional single-enzyme biochemical assays. B) Thiophenol- $\beta$-lac acts as the chemical link between the target enzyme and the secondary enzyme amplifier of DETECT, by selectively eliminating thiophenol upon hydrolysis by E1. Thiophenol subsequently activates E2 by displacing the disulfide-protected cysteine of papain through a thiol-disulfide interchange reaction. Activated papain subsequently generates a signal output by hydrolyzing its probe, BAPA, to produce the chromophore $p$-nitroaniline. 


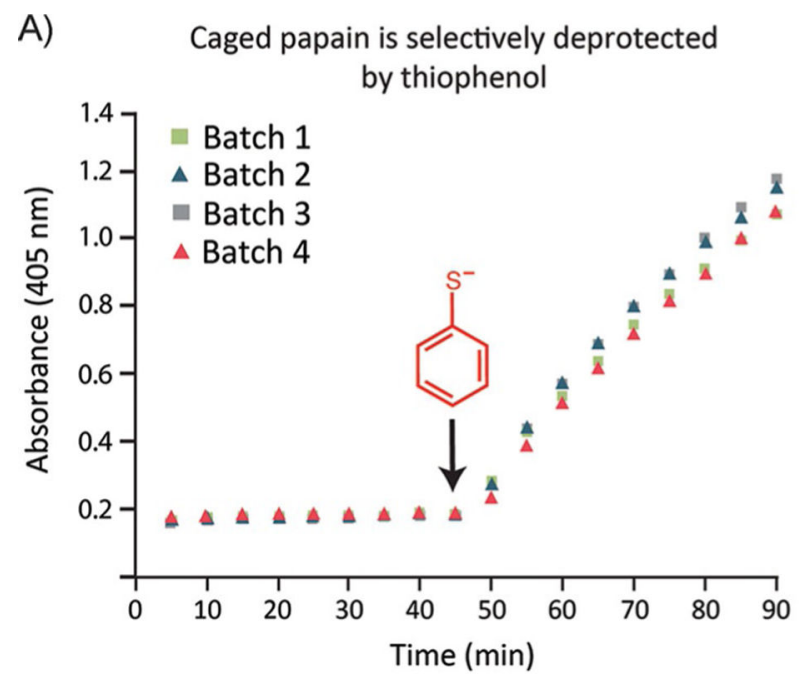

B) Detecting recombinant $\beta$-lactamases

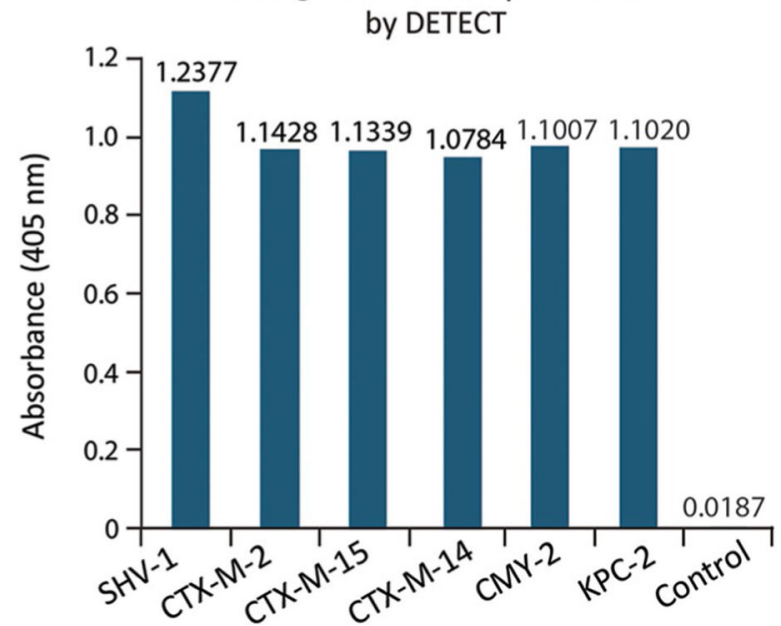

C)

Detection of recombinant TEM-1 activity by DETECT

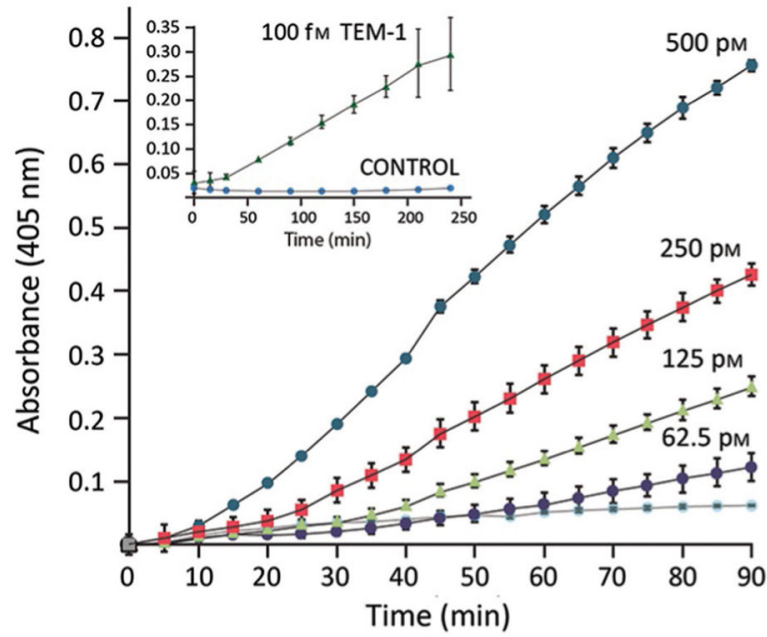

\section{$\beta$-lactamase}

D) Detection of recombinant TEM-1 activity by nitrocefin

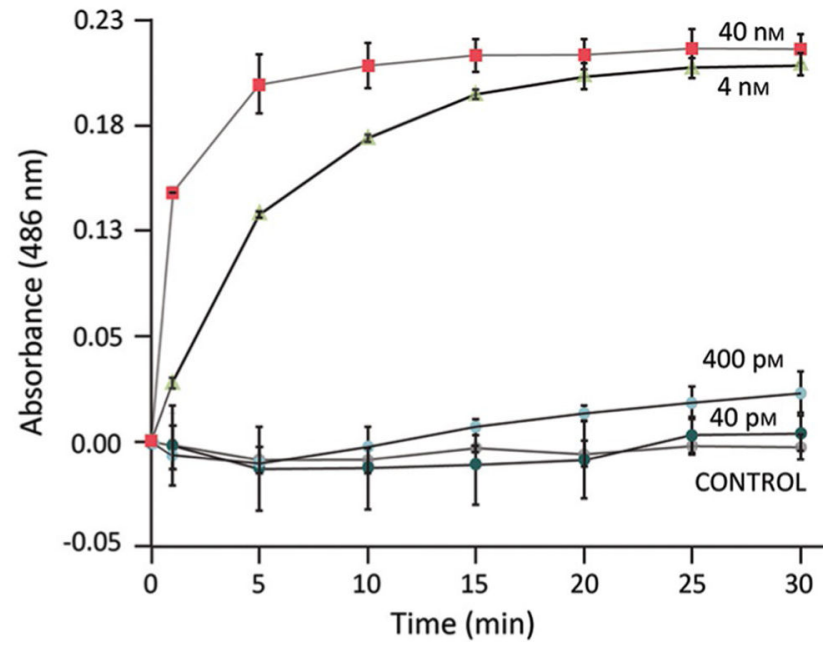

Figure 2.

A) Time-dependent plot demonstrating selective triggering of unique batches of prepared caged papain by thiophenol. B) Plot highlighting the signal outputs observed when $500 \mathrm{~nm}$ of the recombinant $\beta$-lactamases were analyzed by DETECT. The control well contains all components of the DETECT system but no $\beta$-lactamase, so as to account for nonselective hydrolysis of thiophenol- $\beta$-lac or any other nonselective signal generated by the system. $\mathrm{C}$ ) Quantitative time-dependent plot of $\beta$-lactamase activity assays performed with DETECT, in which varied concentrations of recombinant TEM-1 were analyzed. The absorbance at 405 $\mathrm{nm}$ was collected in 5 min intervals and plotted against time. D) Quantitative time-dependent plot of $\beta$-lactamase activity assays performed with a standard $\beta$-lactamase probe, in which varied concentrations of TEM-1 were incubated with nitrocefin $(486 \mathrm{~nm})$. 
A)

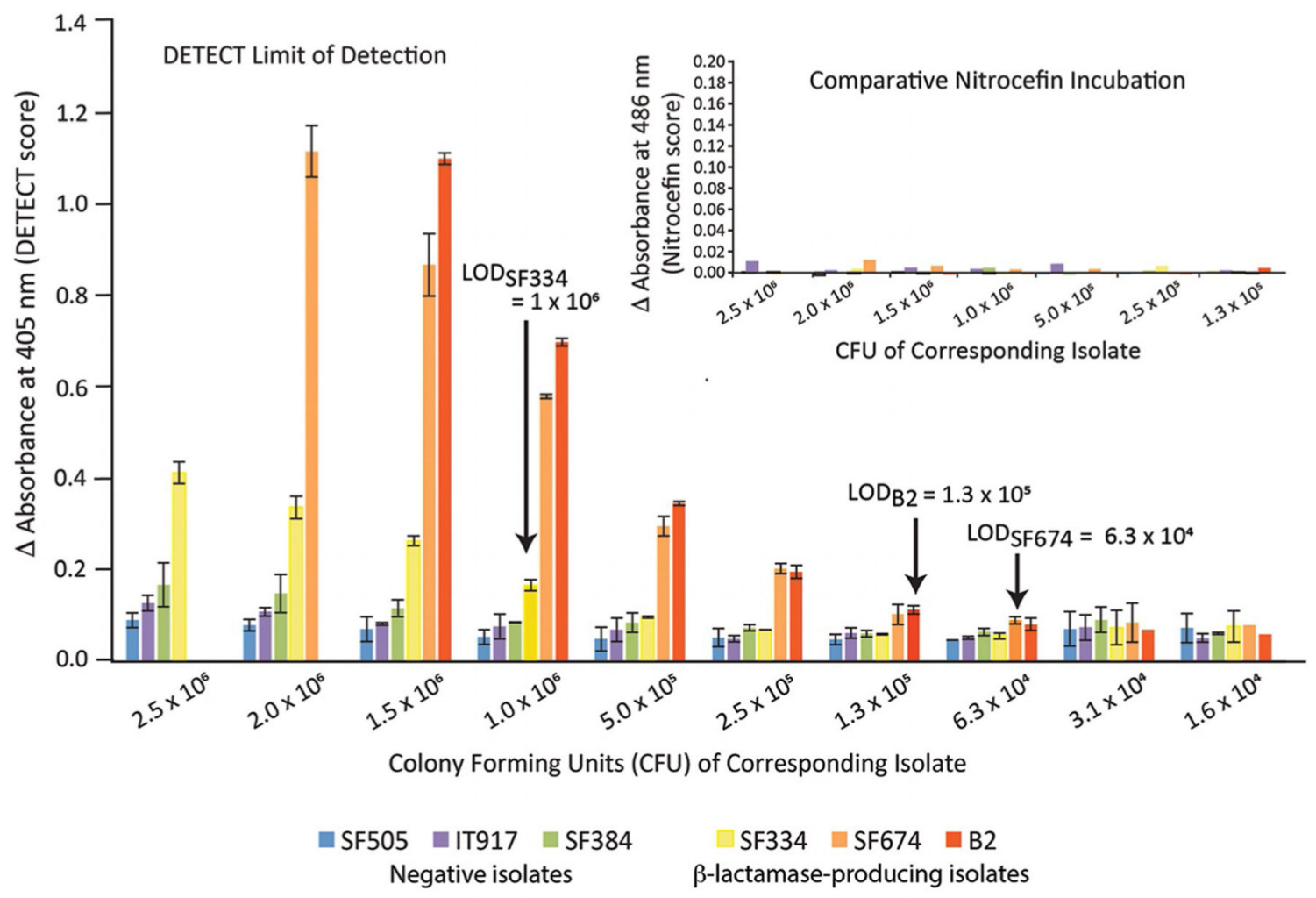

B) DETECT amplifies the limit of detection of standard biochemical assays

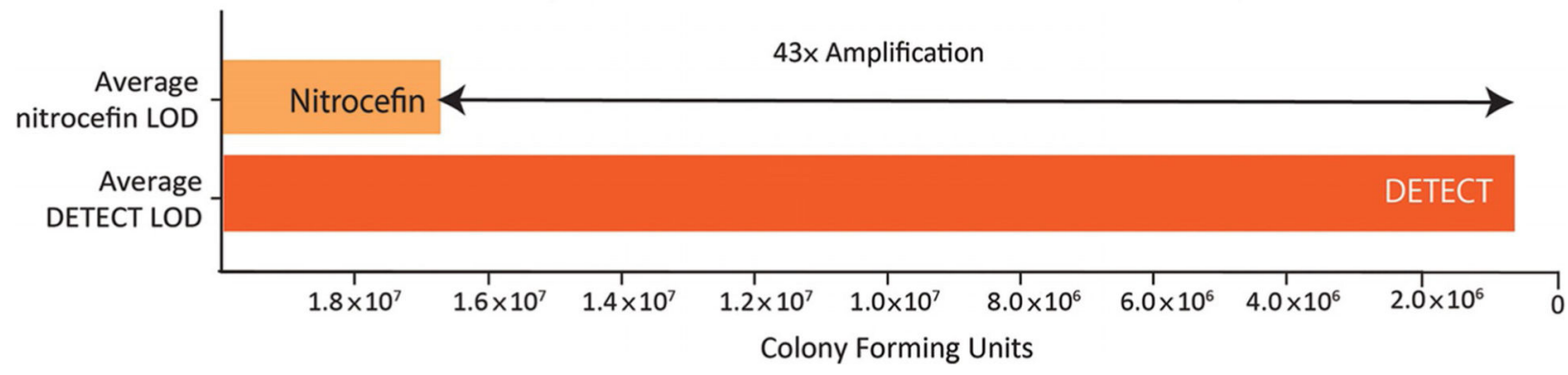

Figure 3.

A) Concentration-dependent plot demonstrating the LOD of DETECT in identifying the activity of $\beta$-lactamase in clinical isolates producing TEM-1, CTX-M-15, or KPC-2. The LODs were determined by comparing the corresponding DETECT scores generated from these samples to the scores from $\beta$-lactam-susceptible isolates. Inset: Concentrationdependent plot highlighting results from the comparative nitrocefin study, in which identical clinical isolate suspensions were tested with the standard chromogenic $\beta$-lactamase probe, nitrocefin. B) Bar plot highlighting the amplification capacity of DETECT compared to nitrocefin. 
A)

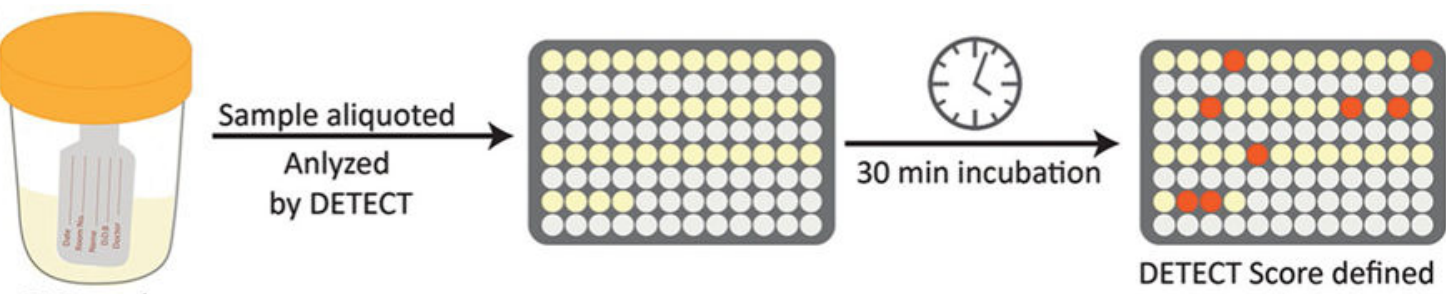

40 Samples

B)

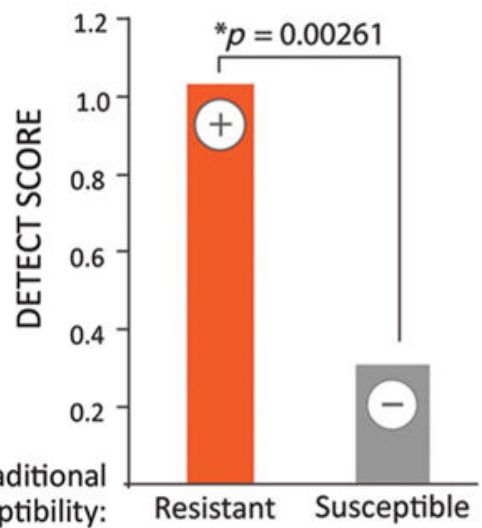

C)

\begin{tabular}{|c|c|c|c|}
\hline \multirow{2}{*}{ DETECT } & \multicolumn{3}{|c|}{ STANDARD } \\
\cline { 2 - 4 } & Amp-Resistant & Amp-Susceptible & SUM \\
\hline+ & 8 & 0 & 8 \\
\hline- & 3 & 29 & 32 \\
\hline SUM & 11 & 29 & 40 \\
\hline
\end{tabular}

${ }^{*}$ Accuracy $=0.925, p=0.01$

*Staistical analysis performed using one-tailed t-test

Figure 4.

A) Schematic overview of the validation study, performed with 40 urine samples collected from patients suspected of having a UTI, with dipstick-positive urines. In this study, $50 \mathrm{~mL}$ of unprocessed urine sample was transferred into a 96-well plate and analyzed by DETECT.

After a 30 min incubation, samples were analyzed with a microplate reader to define the DETECT score. B) Plot demonstrating the difference between the average DETECT score observed in urine samples characterized to contain ampicillin-resistant or ampicillinsusceptible E. coli. Urine samples were categorized as resistant or susceptible based on standard antimicrobial susceptibility testing results ( $p=0.00261$, two-tailed $t$-test). C) Sensitivity and specificity of DETECT when applied as a diagnostic tool to identify $\beta$ lactamase activity as a measure of $\beta$-lactam resistance, where ampicillin resistance was defined as the standard predicate. 